Cite this: J. Mater. Chem. B, 2014, 2, 1400

Received 14th November 2013

Accepted 20th January 2014

DOI: $10.1039 / c 3 t b 21605 e$

www.rsc.org/MaterialsB

\section{Pulsatile drug release from electrospun poly(ethylene oxide)-sodium alginate blend nanofibres $\dagger$}

\author{
Abdessamad Y. A. Kaassis, ${ }^{a}$ Neil Young, ${ }^{\text {b }}$ Naoko Sano, ${ }^{\mathrm{c}}$ Hamid A. Merchant, ${ }^{a}$ \\ Deng-Guang $\mathrm{Yu}^{\mathrm{d}}{ }^{\mathrm{d}}$ Nicholas P. Chatterton ${ }^{* e}$ and Gareth R. Williams*a
}

Novel and highly tuneable pulsatile drug delivery systems have been prepared through the electrospinning of a blend of poly(ethylene oxide) (PEO), sodium alginate (SA), and sodium ibuprofen (SI). The resultant fibres contain crystallites of SI embedded in a PEO-SA matrix, and rather than being obtained as flat mats on the collector plate form novel three dimensional structures extending upwards the needle. Fibres were prepared with a range of loadings of SI and SA. It was found that at $\mathrm{pH} 6.8$ (reminiscent of the intestinal tract) the fibres dissolve very rapidly, freeing all the embedded drug within ca. 20 minutes. However, at $\mathrm{pH} 3$ (representative of the stomach $\mathrm{pH}$ in the fed state or in older patients) an unusual two stage release mechanism is seen. This comprises a rapid burst release, followed by a period where no further drug is released for ca. 120-150 minutes, and then a final stage of release freeing the remainder of the drug into solution. The amount of release in the initial stage, and the length of time between the first and final drug release stages, can be controlled by adjusting the SI and SA contents of the fibres respectively. This results in highly tunable pulsatile release materials.

\section{Introduction}

Electrospinning is a facile top-down technique permitting the fabrication of a wide variety of functional nanocomposites. A solution of a polymer and a functional component is prepared, usually in a volatile solvent, and expelled from a needle (spinneret) towards a metal collector at a controlled rate. A high potential difference is applied between the two and this electrical energy causes rapid evaporation of the solvent, resulting in polymer-based one-dimensional objects with diameters on the nm scale. ${ }^{1-4}$ These fibres have a number of desirable properties (e.g. high surface area) which may be tuned by controlling the processing parameters (voltage applied, needle-to-collector distance, flow rate, solution concentration, etc.). ${ }^{1}$

A large number of studies have reported the use of electrospinning to produce drug-loaded fibres for use as engineered

${ }^{a}$ UCL School of Pharmacy, University College London, 29-39 Brunswick Square, London, WC1N 1AX, UK. E-mail: g.williams@ucl.ac.uk; Tel: +44 (0)207 7535868 ${ }^{b}$ Department of Materials, University of Oxford, Parks Road, Oxford, OX1 3PH, UK ${ }^{c} N E X U S$, Newcastle University, Stephenson Building, Newcastle upon Tyne, NE1 7RU, $U K$

${ }^{d}$ School of Materials Science \& Engineering, University of Shanghai for Science and Technology, Shanghai 200093, China

eSchool of Human Sciences, Faculty of Life Sciences and Computing, London Metropolitan University, 166-220 Holloway Road, London, N7 8DB, UK. E-mail: n.chatterton@londonmet.ac.uk; Tel: +44 (0)207 1334373

$\uparrow$ Electronic supplementary information (ESI) available. See DOI: 10.1039/c3tb21605e drug delivery systems. ${ }^{1,5-9}$ The majority of these studies use onefluid electrospinning, but even in this simple system it is found that variation of the polymer and processing parameters can result in a wide range of drug release behaviours. More recently, more advanced approaches have been employed to develop bespoke drug delivery profiles; these include coaxial electrospinning (using two concentric needles, one nested inside another) and a process combining electrospinning and spraying. ${ }^{10,11}$ The electrospinning of polymer blends has also been explored reasonably widely, and has been applied to drug delivery to achieve a variety of release profiles. ${ }^{\mathbf{1 2 - 1 4}}$ Electrospun materials have successfully been used to modify the rate of drug release,$^{13}$ to target release to a particular part of the body such as the gastrointestinal tract, ${ }^{5}$ or to deliver biphasic or dual-stage drug release. ${ }^{\mathbf{1 0 , 1 1}}$ While such release profiles can be very useful in the development of advanced pharmaceutics, they are inappropriate if a drug has a high first-pass effect or if to be effective it requires very time-specific delivery. ${ }^{15}$ Time-controlled pulsatile release, in which the drug is released after a predefined time period, is more appropriate for such applications.

A number of disease states follow biological rhythms. ${ }^{16}$ For instance, asthma attacks tend to happen mainly late at night, following the body's natural circadian cycle, and pain from rheumatoid arthritis is most intense in the morning. Epilepsy also oscillates in its implications for patients, in response to melatonin secretion. To effectively treat these conditions, precise time-controlled release profiles are required. Pulsatile delivery systems of drugs such as sodium valproate (indicated 
for the treatment of epilepsy) or diltiazem (angina) are hence much sought after to enhance patient compliance and improve clinical outcomes. To date, there are no reports of time-resolved pulsatile release from electrospun nanofibres.

The formation of a system comprising two polymers mixed, with a drug component loaded, offers the potential to deliver novel drug release profiles even from a single fluid electrospinning process. If one of these polymers has $\mathrm{pH}$-sensitive characteristics, then targeted drug release should be possible. One such polymer is alginic acid, commonly available as its sodium salt, which dissolves only at elevated pHs. In order to incorporate sodium alginate (SA) into nanofibres, it is necessary to blend it with a carrier polymer. ${ }^{17} \mathrm{~A}$ number of researchers have developed electrospun fibres containing SA blended with poly(ethylene oxide) (PEO). ${ }^{\mathbf{1 8 - 2 1}}$ Alborzi et al. have used SApectin-PEO fibres to stabilise folic acid,22 and Lee and coworkers have coated poly(vinyl alcohol) fibres with alginate for pH sensitive release. ${ }^{23}$ In 2012 , two sets of researchers independently reported the formation of novel electrospun fibres comprising PEO and SA. ${ }^{\mathbf{2 4 , 2 5}}$ Although both teams used the same building blocks and similar synthetic processes, very different results were obtained: Ma et al. created core-shell nanofibres with a PEO shell and a SA core,$^{25}$ while Bonino and co-workers noted the formation of a "mountain" of fibres on the collector plate instead of the flat mat normally seen, which they attributed to the aggregation of SA on the exterior of the fibres. ${ }^{24}$

It is clear therefore that blended PEO-SA fibres have interesting properties from an academic viewpoint; in addition, PEO is a highly suitable polymer for drug delivery systems because it is classified by the FDA as a "generally regarded as safe" excipient, and since it is hydrophilic can aid the solubilisation of poorly water soluble drugs. Variation in the molecular weight of the polymer can result in tuning of the drug release properties from PEO composites. ${ }^{26}$ Furthermore, SA has been exploited for many years as a pH-responsive drug release system. ${ }^{27-29}$ In this work, these properties were exploited to develop drug delivery systems based on PEO-SA blend fibres, with sodium ibuprofen employed as a model drug.

\section{Experimental}

\section{Electrospinning}

Fibres were prepared by co-dissolving PEO (Sigma; MW ca. 400000 ) and SA (Fisher; MW 120 000-190 000) in deionised water to give final concentrations of $5 \%$ and $1.5 \% \mathrm{w} / \mathrm{v}$ respectively. $10 \mathrm{~mL}$ of this solution was then combined with varied amounts of sodium ibuprofen (SI; Sigma-Aldrich; 98\%). Analogous solutions were prepared with varied SA concentrations. This resulted in a series of spinning solutions as detailed in Table 1.

Each solution was carefully loaded into a $5 \mathrm{~mL}$ syringe, with great care taken to avoid any air bubbles. The syringe was fitted with a metal needle tip (spinneret; internal diameter $0.84 \mathrm{~mm}$ ), and the positive electrode of a high voltage DC power supply (HCP 35-65000, Fug Elektronik, Rosenheim, Germany) connected to the spinneret. The grounded electrode was connected to a metal collector $\left(17 \times 17 \mathrm{~cm}^{2}\right)$ wrapped with aluminium foil. The humidity of the spinning chamber was maintained below
Table 1 The compositions of the fibres prepared in this study. $10 \mathrm{~mL}$ of a $5 \% \mathrm{w} / \mathrm{V}$ PEO-SA solution was added to the amount of ibuprofen detailed below to prepare spinning solutions

\begin{tabular}{|c|c|c|c|c|c|}
\hline $\begin{array}{l}\text { Fibre } \\
\text { ID }\end{array}$ & $\begin{array}{l}\text { Mass SI } \\
(\mathrm{g})\end{array}$ & $\begin{array}{l}\text { SA conc. } \\
(\% \mathrm{w} / \mathrm{v})\end{array}$ & $\begin{array}{l}\% \text { PEO } \\
\text { in fibre } \\
(w / w)\end{array}$ & $\begin{array}{l}\% \mathrm{SA} \text { in } \\
\text { fibre } \\
(\mathrm{w} / \mathrm{w})\end{array}$ & $\begin{array}{l}\% \text { SI in } \\
\text { fibre } \\
(w / w)\end{array}$ \\
\hline F0 & 0 & 1.5 & 76.9 & 23.1 & 0 \\
\hline F1 & 1 & 1.5 & 30.3 & 9.1 & 60.6 \\
\hline F2 & 0.2 & 1.5 & 58.8 & 17.6 & 23.5 \\
\hline F3 & 0.1 & 1.5 & 66.7 & 20.0 & 13.3 \\
\hline F4 & 1 & 1 & 31.3 & 6.3 & 62.5 \\
\hline F5 & 1 & 2 & 29.4 & 11.8 & 58.8 \\
\hline F6 & 1 & 2.5 & 28.6 & 14.3 & 57.1 \\
\hline
\end{tabular}

$20 \%$, and its temperature at $37 \pm 2{ }^{\circ} \mathrm{C}$. The feed rate was maintained at $0.5 \mathrm{~mL} \mathrm{~h}^{-1}$ using a syringe pump (KDS100, ColeParmer, Vernon Hills, IL, USA). The spinneret to collector distance was $13 \mathrm{~cm}$ and the applied voltage $20 \mathrm{kV}$.

\section{Characterisation}

Fibre morphology and composition. The fibres were visualised using scanning electron microscopy on a JSM-5600LV instrument (JEOL, Tokyo, Japan) or a Quanta 200 FEG ESEM (FEI, Hillsborough, OR, USA). Samples were gold sputtered prior to examination. The images obtained were analysed using the ImageJ software (National Institutes of Health, Bethesda, MD, USA); fibre diameters were measured at over 50 locations and these data used to determine their average size. The results are reported as mean \pm S.D. Scanning transmission electron microcopy was additionally performed on selected samples with the aid of a JEM-3000F HR (S)TEM (JEOL, Tokyo, Japan) operating at $300 \mathrm{kV}$. X-ray photoelectron spectroscopy was performed at the NEXUS facility (Newcastle University). A K-alpha instrument (Thermo Scientific, East Grinsted, UK) equipped with a monochromated $\mathrm{Al} \mathrm{K} \alpha \mathrm{X}$-ray source was used with a pass energy of $40 \mathrm{eV}$ and step size of $0.1 \mathrm{eV}$. Spectra were processed using the CasaXPS software (Casa Software Ltd., Teignmouth, UK).

The structure of the SI post-electrospinning was probed by nuclear magnetic resonance (NMR). Spectra were recorded at ambient temperature, on an Avance-500 instrument $\left({ }^{1} \mathrm{H}\right.$ frequency: $500.13 \mathrm{MHz}$; Bruker, Karlsruhe, Germany). Samples were dissolved in $\mathrm{D}_{2} \mathrm{O}$ prior to measurement. The drug loading in the fibres was determined using high-performance liquid chromatography (HPLC; 1260 Infinity instrument, Agilent, Santa Clara, CA, USA). The stationary phase comprised a Supleco® Discovery HS F5 HPLC column ( $5 \mu \mathrm{m}$ particle size, 150 $\times 4.6 \mathrm{~mm}$ ) and the mobile phase was acetonitrile- $0.1 \%$ TFA. Elution was carried out isocratically $(74: 26)$ with a flow rate of $1.0 \mathrm{~mL} \min ^{-1}$ and injection volume of $10 \mu \mathrm{L}$. The SI concentration was assayed at $264 \mathrm{~nm}$ at room temperature.

\section{Physical form and component interactions}

The physical form of the components in the fibres was probed by diffraction and calorimetry. Powder X-ray diffraction (XRD) was undertaken with a MiniFlex 600 diffractometer (RigaKu, 
Tokyo, Japan) using $\mathrm{Cu} \mathrm{K} \alpha$ radiation at $40 \mathrm{kV}$ and $15 \mathrm{~mA}$. Differential scanning calorimetry was performed with the aid of a DSC Q2000 instrument (TA Instruments, New Castle, DE, USA). Samples were heated from 25 to $150{ }^{\circ} \mathrm{C}$ at $10{ }^{\circ} \mathrm{C} \mathrm{min}{ }^{-1}$ under a flow of nitrogen $\left(50 \mathrm{~mL} \mathrm{~min}^{-1}\right)$.

The interactions between the components in the fibres were explored by IR spectroscopy (Spectrum 100 spectrometer, Perkin Elmer, Waltham, MA, USA) over the range $650-4000 \mathrm{~cm}^{-1}$ with a resolution of $1 \mathrm{~cm}^{-1}$.

\section{Drug release}

0.15-0.4 $\mathrm{g}$ (depending on SI loading) of the fibre structure was immersed in $1 \mathrm{~L}$ of a release fluid (either a $\mathrm{pH} 3$ acetic acid solution, or a phosphate buffer at $\mathrm{pH} \mathrm{6.8)}$ and stirred at $50 \mathrm{rpm}$ and $37{ }^{\circ} \mathrm{C}$ on a dissolution apparatus (SR8 Plus, Hanson Research, Chatsworth, CA, USA). Aliquots were removed at regular intervals and the SI content determined by UV spectroscopy (UV1800, Shimadzu, Kyoto, Japan) at $264 \mathrm{~nm}$. All release studies were performed under sink conditions.

\section{Results and discussion}

\section{Fibre morphology}

A photograph of the $\mathrm{F} 1$ fibres $(60.6 \% \mathrm{SI} w / \mathrm{w})$ is given in Fig. 1(a). It is immediately clear that the fibres form a three-dimensional "mountain" structure rather than a flat mat on the collector plate: our results thus agree with those of Bonino et al. ${ }^{24}$ Scanning electron microscopy images were recorded on samples taken from different points of the 3D fibre structure (base, middle, and tip); data for the base and tip are included in Fig. 1. The SEM data show the fibres throughout the 3D structure to have smooth and uniform structures. There is no obvious "bead-on-string" morphology visible in any of the images. Each fibre appears to have a constant diameter, although there seems to be a difference in size between the top and bottom of the structure (318 \pm 53 and $233 \pm 44 \mathrm{~nm}$ respectively). Looking at the outside of the structure, there are no visible particles; however, bisection through the centre reveals a number of small particles inside the structure at its apex (see Fig. 1(e)). The F2 and F3 fibres (23.5 and $13.3 \%$ SI w/w respectively) exhibited analogous features, with F2 having diameters of $271 \pm 42 \mathrm{~nm}$ (top) and $209 \pm 48 \mathrm{~nm}$ (base) and F3 sizes of $260 \pm 42 \mathrm{~nm}$ (top) and $205 \pm 53 \mathrm{~nm}$ (base). There thus appears to be a decline in fibre diameter as the SI content is reduced.

The observation of core-shell structure by $\mathrm{Ma}^{25}$ led us to record scanning transmission electron microscopy (STEM) images of the fibres (Fig. 1(f)). No core-shell structure is visible in any of the fibres, but there are regions of high- and lowcontrast present throughout the fibres.

$\mathrm{X}$-ray photoelectron spectroscopy (XPS) was invoked to probe the nature of the fibre surfaces further. XPS spectra of the starting materials (SI, SA, and PEO) were recorded, as were the spectra of the fibre materials. The $\mathrm{C} 1 \mathrm{~s}$ spectra of the fibres were then peak fitted using the $\mathrm{C} 1 \mathrm{~s}$ spectra of the starting materials as references to determine the relative amounts of each material at the fibre surfaces. The results of this analysis are given in Table 2. The data for the blank F0 (PEO-SA only) fibres show that the surface comprises almost entirely PEO, in agreement with the results observed by Ma et al., ${ }^{25}$ although even with these fibres we see no core-shell structure by STEM. The fractions of the surface comprising SI are, within the error of the measurements, identical to those calculated from the weight ratios of the components. However, for F1-F3 the SA fraction at the surface is greater than that calculated from the starting material weight ratios, while the PEO percentage is lower.

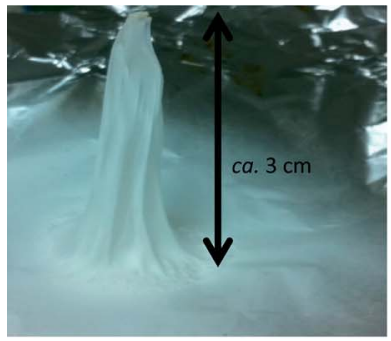

(a)

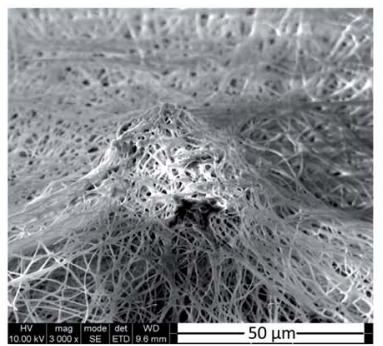

(d)

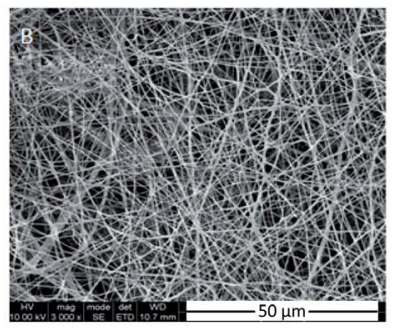

(b)

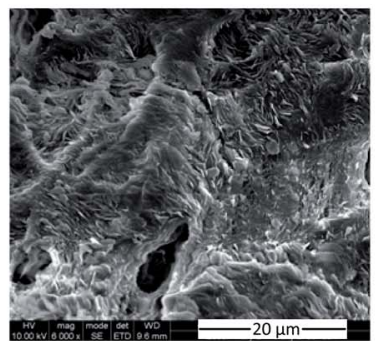

(e)

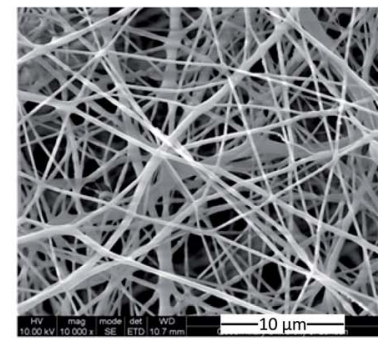

(c)

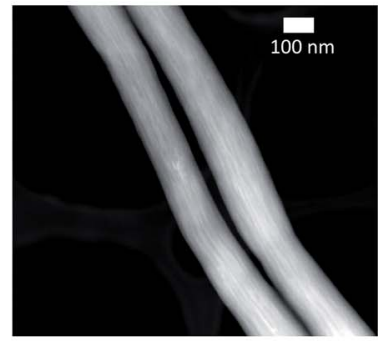

(f)

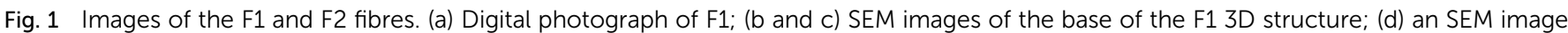

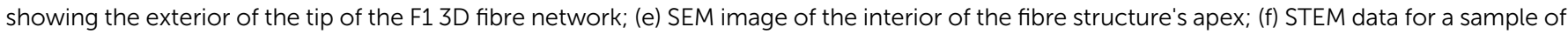
F2 collected on a lacey $C$ coated Cu grid. 
Table 2 The ratios of PEO, SA, and SI at the surface of the fibres, calculated from XPS data. Samples were taken from the top of the 3D fibre structure

\begin{tabular}{lllrl}
\hline & & \multicolumn{3}{l}{ Fractional composition (\%) } \\
\cline { 3 - 5 } Fibre ID & $\begin{array}{l}\text { \% SI } \\
\text { in fibre }(w / w)\end{array}$ & \multicolumn{1}{l}{ PEO } & \multicolumn{1}{c}{ SA } & SI \\
\hline F0 & 0 & $99.3 \pm 1.1$ & $0.7 \pm 1.1$ & - \\
F1 & 60.6 & $15.0 \pm 1.2$ & $24.0 \pm 2.0$ & $60.3 \pm 3.0$ \\
F2 & 23.5 & $37.0 \pm 1.0$ & $38.7 \pm 1.9$ & $24.3 \pm 1.5$ \\
F3 & 13.3 & $49.0 \pm 7.8$ & $39.0 \pm 9.0$ & $12.0 \pm 2.5$ \\
\hline
\end{tabular}

Although no core-shell structure could be seen by STEM, the XPS data indicate that there is some aggregation of SA at the fibre surface as proposed by Bonino et al..$^{\mathbf{2 1 , 2 4}}$

\section{$X$-ray diffraction}

X-ray diffraction (XRD) patterns for samples taken from the top and bottom of the F1 and F2 structures are given in Fig. 2, together with patterns for the starting materials. PEO is a crystalline polymer, and hence shows two distinct reflections at 19 and $23^{\circ}$. SI is also crystalline, and exhibits numerous reflections. SA is amorphous (see ESI, Fig. S1†). Both the top and base of the F1 structure show the presence of a number of Bragg reflections (see Fig. 2(a)). These appear to occur at approximately the same positions as the SI and PEO reflections, although the reflections are considerably broadened and also shifted to higher angle. The increased broadness of the SI reflections is consistent with the presence of SI crystallites with reduced size ( $c f$. the pure drug) in the fibre structure. This is also seen in the SEM data; the size of the particles which can be observed in Fig. 1(e) is found to be $c a .1295 \pm 229 \mathrm{~nm}$. Prior to dissolution and electrospinning, the SI particles had sizes of approximately $3 \pm 1.5 \mu \mathrm{m}$ and hence there has been a significant reduction in particle size as a result of the processing. The reason for the shift to higher angle is more puzzling, but is tentatively assigned to the possible removal of defects in the SI structure and/or changes in the stress within the particles as a result of the electrospinning process. The reflections corresponding to PEO also shift. This must be a result of the incorporation of SA and SI into the PEO lattice, causing its parameters to change. The XRD patterns for F2 and F3 also show shifts in the peak positions of PEO (see Fig. 2(b) for data on F2), although the reflections corresponding to SI can no longer be clearly resolved as a result of the reduction in its content.

\section{Differential scanning calorimetry}

Differential scanning calorimetry (DSC) data confirm the presence of crystalline SI and PEO in the fibres. DSC traces of the raw materials reveal melting endotherms, for SI at $100.3^{\circ} \mathrm{C}$ and for PEO at $69.3^{\circ} \mathrm{C}$. Data for F1 and F2 can be found in Fig. 3. The traces exhibit a sharp endotherm at $62-63{ }^{\circ} \mathrm{C}$, attributed to the melting of PEO. It is thus clear that the PEO retains its crystalline nature upon incorporation into fibres. A second sharp endotherm is visible at $92.5-93.5{ }^{\circ} \mathrm{C}$; this latter feature is superimposed on a broad endotherm with an apex at ca. 105$110{ }^{\circ} \mathrm{C}$. These two features are respectively believed to correspond to the SI melting peak, and the loss of water (incomplete evaporation of the water solvent used for electrospinning will result in some being incorporated into the fibres). SA melts at $T>300{ }^{\circ} \mathrm{C}$, and hence is not seen in the DSC traces.

There are no significant differences in the DSC behaviour of the top and bottom of the fibre structures. The presence of very distinct SI and PEO melting endotherms confirms both to be present in a crystalline form throughout the materials.

\section{FTIR spectroscopy}

FTIR spectra for F1, PEO, SA and SI are included in Fig. 4, together with the chemical structures of the constituent components of the fibres. The spectrum of SI contains distinct peaks around $3000-3500 \mathrm{~cm}^{-1}$ (H-bonded $\mathrm{OH}$ stretches, arising from the presence of some water in the material), 3000$2850 \mathrm{~cm}^{-1}$ (alkane $\mathrm{C}-\mathrm{H}$ stretches), and characteristic peaks at 1545 and $1408 \mathrm{~cm}^{-1}$ (respectively asymmetric and symmetric carboxylate stretches). The SA spectrum also shows a broad band from $c a .3700-2850 \mathrm{~cm}^{-1}$, corresponding to $\mathrm{H}$-bonded $\mathrm{OH}$ groups and $\mathrm{C}-\mathrm{H}$ stretches. Carboxylate peaks can be seen at 1589 and $1403 \mathrm{~cm}^{-1}$, and $\mathrm{C}-\mathrm{O}$ vibrations are at $1020 \mathrm{~cm}^{-1}$. The PEO spectrum has a distinct peak at $c a .2875 \mathrm{~cm}^{-1}$ arising from $\mathrm{C}-\mathrm{H}$ stretches, and the $\mathrm{C}-\mathrm{O}$ stretch is centred around $1095 \mathrm{~cm}^{-1}$. The spectra recorded from the top and bottom of F1

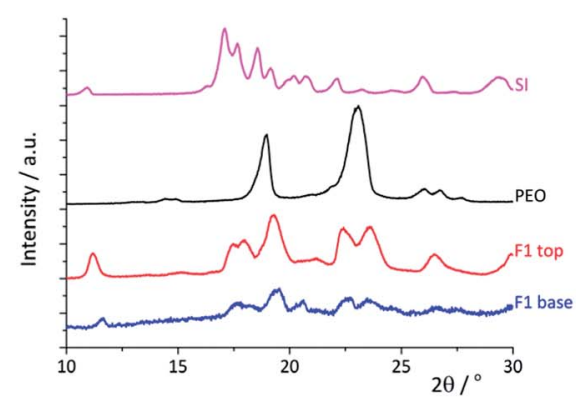

(a)

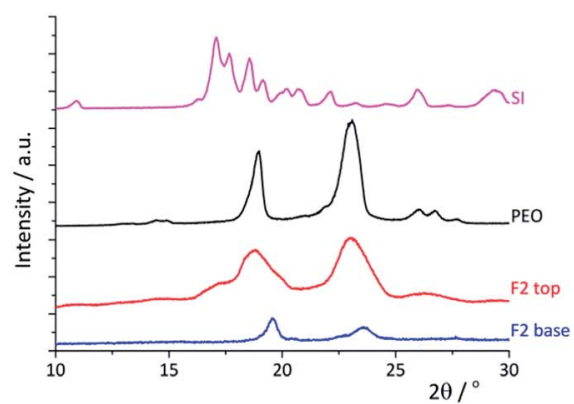

(b)

Fig. 2 XRD patterns of the top and base of (a) the F1 structure $(60.6 \% \mathrm{SI})$ and (b) F2 (23.5\% SI), together with those of PEO and SI. SA was found to be amorphous by X-ray diffraction (see Fig. S1 $\uparrow$ ). 


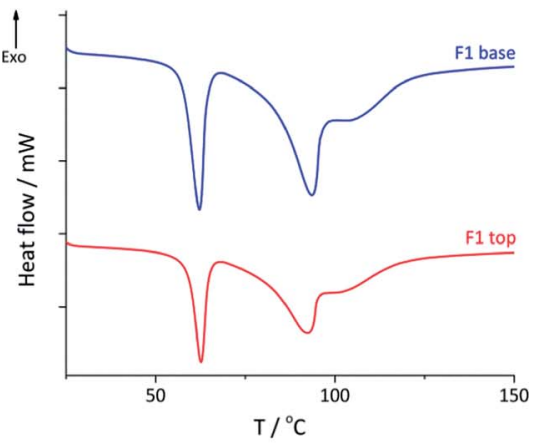

(a)

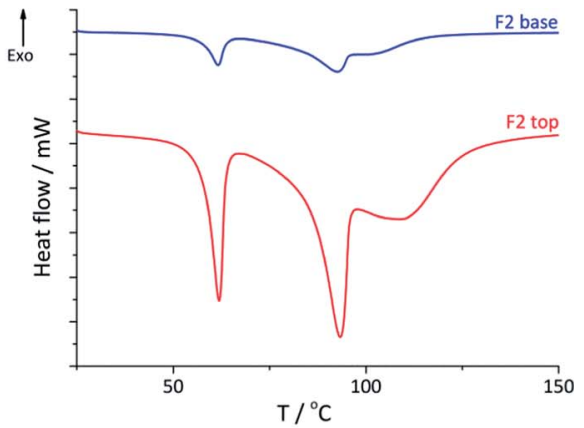

(b)

Fig. 3 DSC traces of the top and base of (a) the F1 structure $(60.6 \% \mathrm{SI})$, and (b) the F2 structure $(23.5 \% \mathrm{SI})$.

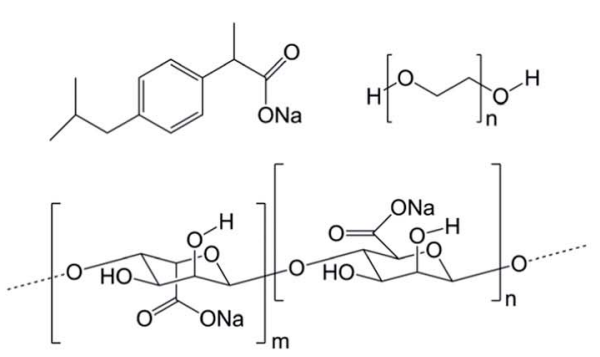

(a)

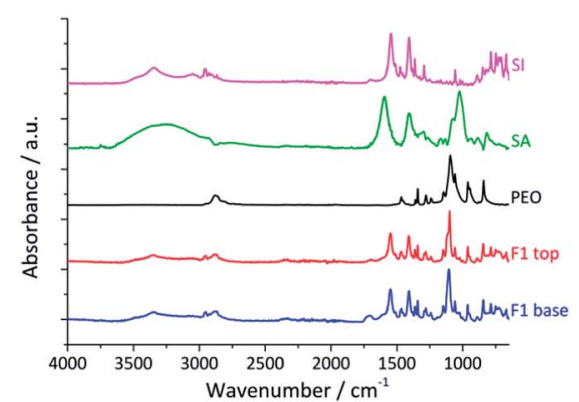

(b)

Fig. 4 (a) The chemical structure of the materials used in this study [top left: SI; top right: PEO; bottom: SA], and (b) IR spectra of the F1 fibres and associated starting materials.

are essentially identical, and contain features from each of the component species. A broad feature from $3500-3000 \mathrm{~cm}^{-1}$ arises from $\mathrm{H}$-bonded $\mathrm{OH}$ groups, and peaks at 2950 and $2875 \mathrm{~cm}^{-1}$ from the SI and SA alkyl C-H bonds. The SI carboxylate groups are visible at 1549 and $1408 \mathrm{~cm}^{-1}$. Although the latter has not moved, the asymmetric stretch has shifted in wavenumber, indicating the existence of interactions between $\mathrm{SI}$ and the other components of the fibres. The PEO C-O stretch is seen at $1095 \mathrm{~cm}^{-1}$.

Overall, therefore, the IR data indicate the successful incorporation of all three components into the fibres, and suggest that SI is interacting with the other components, most likely through the formation of H-bonds. The IR spectra of the other materials show largely analogous features, confirming the successful formulation of fibres containing all three components intact.

\section{NMR spectroscopy and drug loading}

NMR spectroscopy was performed on samples of the fibre mats dissolved in $\mathrm{D}_{2} \mathrm{O}$. The spectra after dissolution are observed to contain all the features of the SI starting material (see ESI, Fig. S2 $\dagger$ ), confirming that the structural integrity of the drug molecule is retained after electrospinning. The drug loading into the fibres was also verified by HPLC, and found to be $100 \%$ within the error of the measurement (for F1, the values observed were $101 \pm 2.1 \%$ for the top of the fibre structure and $99 \pm 1.7 \%$ for the base).

\section{Drug release studies}

Since the SI remains intact after spinning, the fibre structures may have utility as drug delivery systems. Drug release was studied in two different $\mathrm{pH}$ milieu: a pH 3 acetic acid solution (representative of the $\mathrm{pH}$ at the boundary between the fundic and lower stomach, and typical of stomach $\mathrm{pH}$ in the fed state or in the elderly) and a $\mathrm{pH} 6.8$ phosphate buffer mimicking the small intestine. 0.15-0.4 $\mathrm{g}$ (depending on SI loading) of the fibre structure was immersed in $1 \mathrm{~L}$ of the release fluid and stirred at $50 \mathrm{rpm}$ and $37^{\circ} \mathrm{C}$. Aliquots were removed at regular intervals and the SI content determined by UV spectroscopy. The results are depicted in Fig. 5 .

At pH 3, a highly unusual two-stage pulsatile drug release profile was observed: to the best of our knowledge, this has never before been seen for electrospun nanofibre systems. Within the first 5-10 $\mathrm{min}$ after the fibres are added to the release medium, there is a very rapid burst release of drug. The amount of drug released in this stage is dependent on the SI content of the fibres, with a greater burst release being seen as the SI loading increases. There is then a plateau in which almost no further drug is released for 85-120 min, after which SI is freed from the fibres at an almost constant rate over a further 50-200 min, depending on the fibre loading. The F1-F3 fibres thus comprise time-controlled pulsatile drug delivery systems, where there is an "on/off" pattern of drug release (bursts of release with a lag period between them). 


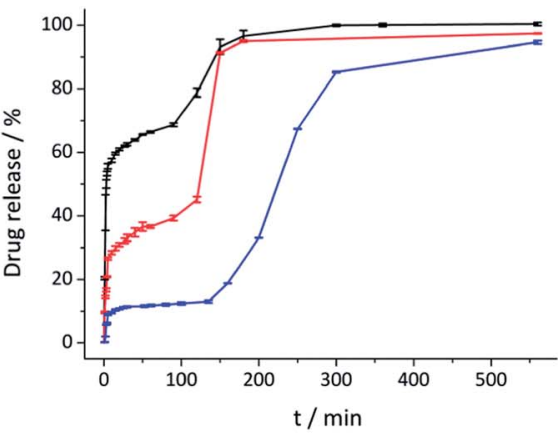

(a)

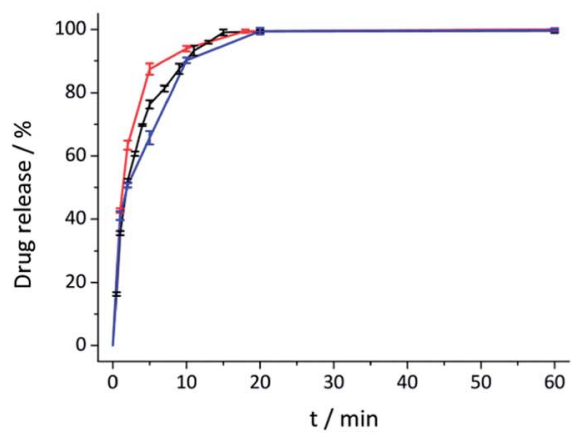

(b)

Fig. 5 Ibuprofen release from the nanofibres at (a) pH 3 and (b) pH 6.8. Fibres F1 (-), F2 ( - ) and F3 ( - ) are shown; these contain respectively 60.6 , 23.5 , and $13.3 \% \mathrm{w} / \mathrm{w}$ SI. Three independent experiments were performed (each with a separate fibre mat), and results are reported as mean \pm S.D.

In contrast, in the pH 6.8 buffer, rapid SI release over $c a .15$ $\min$ is seen, with essentially identical release profiles regardless of the SI loading in the fibres. $\mathrm{pH} 3$ is typical of stomach $\mathrm{pH}$ when in the fed state. By appropriate selection of the fibre composition it would be possible to deliver a desired loading dose of drug in the stomach, with the remainder retained in the fibres for longer than the typical stomach transit time $(2 \mathrm{~h})$ and releasing rapidly upon entry to the intestinal tract. The percentage of SI released in the first stage is approximately the same as the w/w percentage of SI in the fibres, meaning it would be facile to design materials to release a given amount of SI in the stomach. The formulation acts as a dual trigger release system, and can be activated by both $\mathrm{pH}$ and time. This could provide a failsafe approach for pulsatile release in patients with significantly longer gastric residence times, or where the intestinal $\mathrm{pH}$ is not high enough for the formulation to release the second part of its drug loading. In such cases the drug will still be released after a time delay, which can be tuned into the formulation to achieve the desired pharmacokinetic profile.

Given that the extent of burst release can be tuned by varying the SI content, experiments were also performed in which the content of SA in the fibres was varied. F1 contains $9.1 \% \mathrm{SA} w / \mathrm{w}$, and materials were prepared with the same amount of SI in the starting solutions, but yielding products with $6.3,11.8$, and $14.3 \% \mathrm{SA} \mathrm{w} / \mathrm{w}(\mathrm{F} 4,5$, and 6 respectively; see Table 1$)$. In all cases, $3 \mathrm{D}$ "mountain" structures were deposited on the collector plate. The release profiles obtained at $\mathrm{pH} 3$ are given in Fig. 6 . The initial burst release is observed to be $c a$. $60 \%$ for all SA contents (approximately the same as the SI loading in the fibres), but with increasing SA content the second phase of release is retarded. With $6.3 \% \mathrm{SA}(\mathrm{F} 4)$ the plateau lasts for some $45 \mathrm{~min}$, whereas with $14.3 \% \mathrm{SA}$ (F6) it is greater than 7 hours, and even after 3 days of release only around $75 \%$ of the embedded drug was released. It is therefore possible to exert very precise control over the drug release characteristics: the SI content determines the amount of drug released in the first "burst" release, while the SA content dictates the second stage of release.

\section{Drug release mechanism}

The trends identified above can be explained by a simple model. The STEM data indicate that there are regions of the fibres

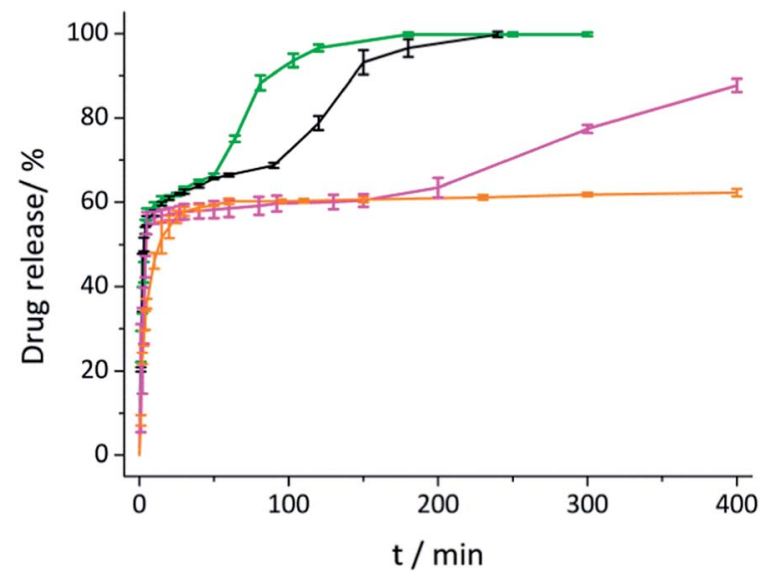

Fig. 6 Drug release from fibres with varied SA contents at $\mathrm{pH}$ 3: F4 (6.3\% SA; -); F1 (9.1\% SA; -); F5 (11.8\% SA; -); and, F6 (14.3\% SA; -). For clarity, data are shown out only to $400 \mathrm{~min}$, although experiments were performed for up to three days. Three independent experiments were performed (each with a separate fibre mat), and results are reported as mean \pm S.D.

which are relatively high contrast, and others where less contrast is present. This suggests that there may be some areas of the fibres which are more SA rich than others. SI particles are also seen to be present at the fibre surfaces by SEM. PEO is freely soluble at $\mathrm{pH} 3$, while SA is not. The dissolution experiments
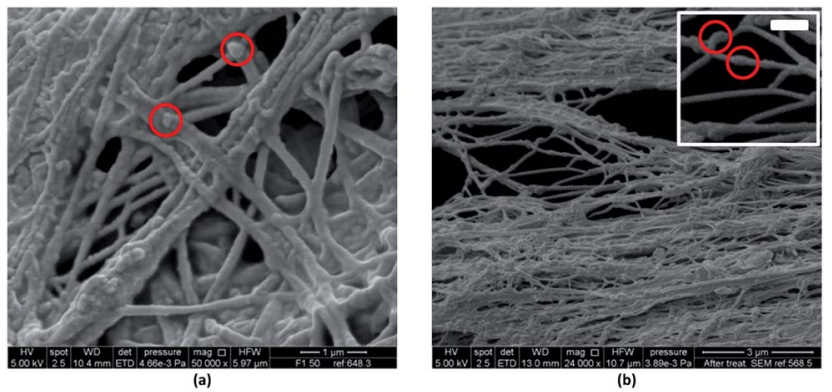

Fig. 7 SEM images of F1 fibres exposed to a $\mathrm{pH} 3$ environment for $10-$ 20 minutes. (a) The fibre mat after immersion in a few drops of acidic medium; (b) the mat after suspension in $1 \mathrm{~L}$ of medium. The inset in (b) is an enlargement of a section of the main image, and the scale bar represents $500 \mathrm{~nm}$. 


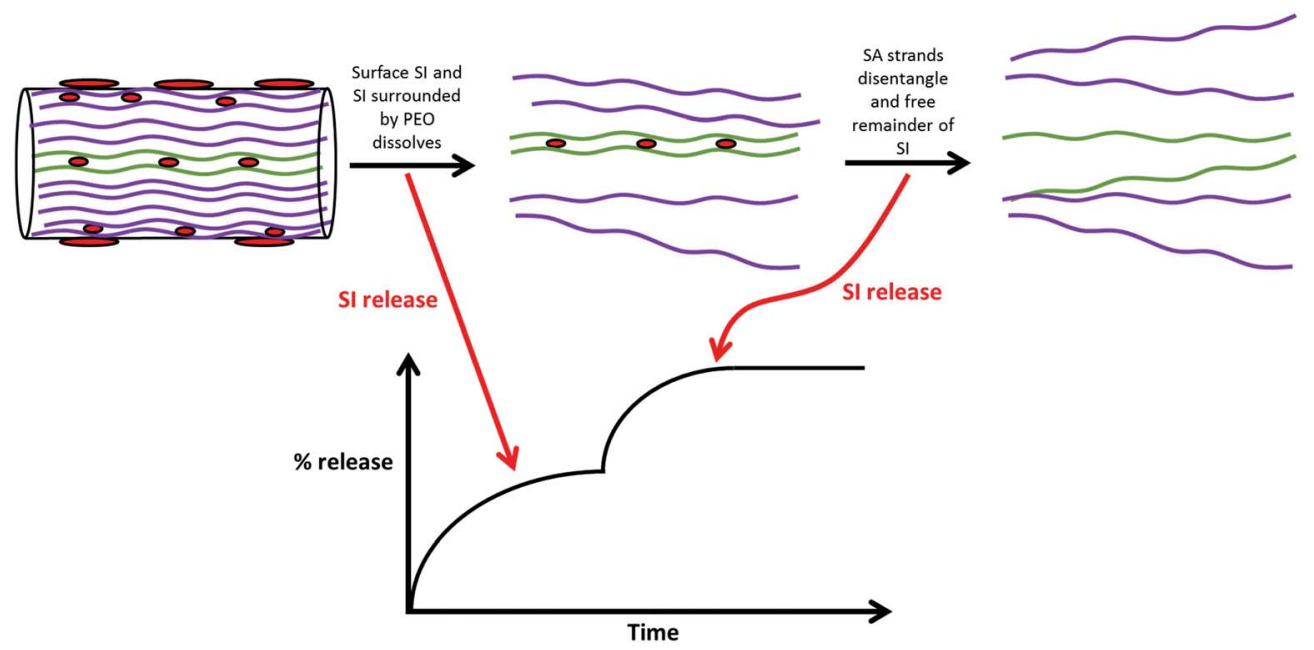

Fig. 8 A schematic illustrating the proposed mechanism responsible for the two-stage pulsatile drug release observed at $\mathrm{pH} 3$.

were performed under sink conditions, and hence the SI will also be freely soluble. We can therefore hypothesise that SI particles on the surface, and also SI ion pairs at the fibre edges, are freed into solution very rapidly at this low $\mathrm{pH}$. At the same time the PEO molecules will disentangle and begin to dissolve, freeing the SI linked to PEO through an erosion mechanism. The greater the SI content, the more SI which exists as particles or near the surface, and hence the larger the percentage of drug released in the initial burst phase.

The SA will be insoluble at $\mathrm{pH} 3$, and so its strands might be expected to cluster together while the other components of the fibre dissolve. Similar results have been reported in the literature: SA is known to aggregate at low pH (ca. 3) into core-shell micelle-like structures. ${ }^{30}$ The different residues in SA have different $\mathrm{p} K_{\mathrm{a}}$ values (3.38 and 3.64 respectively for the $\beta$-Dmannuronic and $\alpha$-L-guluronic acids ${ }^{31}$ ), which results in some portions of its polymer chain becoming hydrophobic and others hydrophilic at this low pH. We hypothesised that such effects might also be involved in the fibre systems. To explore this, F1 fibres were exposed to the $\mathrm{pH} 3$ medium for 10-20 minutes (sufficient time for the first release stage to be complete), recovered by filtration, and assessed by SEM. Experiments were performed both in which the fibres were immersed in $1 \mathrm{~L}$ of the $\mathrm{pH} 3$ solution, and where they were treated with only a few drops of this solution. The results are given in Fig. 7 .

The images shows that upon addition of the $\mathrm{pH} 3$ medium, the fibres swell (Fig. 7(a)) and begin to dissolve and disentangle (Fig. 7(b)) as the PEO absorbs water. In the centre of the image in Fig. 7(a), where individual fibres can be resolved, the fibre diameter is of the order of $140-250 \mathrm{~nm}$, somewhat smaller than the initial material. Upon exposure to a larger volume of the acidic medium (Fig. 7(b)), the diameter declines further, to around $100 \mathrm{~nm}$. Small particles can also be seen in both images. Although it is uncertain exactly what the nature of the particles visible in Fig. 7 is (they may be crystals of neutral ibuprofen or possibly undissolved SI, although the latter is unlikely in Fig 7(b) where the experiment was performed in sink conditions and the SI will be highly soluble) it is thought that SA aggregates also form from the PEO-SA-SI fibres, whether they be micellelike or larger linear aggregates reminiscent of the original fibre structure. The low solubility of SA at pH 3 means that SI in SA-rich areas of the fibres is hindered in dissolution. However, because the fibres comprise blends of PEO and SA, with PEO present in greater amounts, it appears that the swelling and dissolution of the PEO upon prolonged exposure to the acetate solution is sufficient to cause the SA strands eventually to separate sufficiently to free the SI associated with them, at least with lower SA contents. Increased SA contents result in the formation of more robust aggregates, and hence as this increases so does the time before which the final stage of drug release begins.

This model is consistent with all the observed results, and is depicted in Fig. 8. At pH 6.8 both PEO and SA are freely soluble, and so the fibre mat dissolves completely, releasing all the embedded SI very rapidly.

\section{Conclusions}

This paper reports for the first time blended fibres containing PEO, sodium alginate and sodium ibuprofen. The as-formed fibre aggregates exhibit novel 3D network structures, and at $\mathrm{pH}$ 3 two-stage pulsatile drug release is seen. The release profile may be precisely tuned by varying the sodium alginate and sodium ibuprofen contents of the fibres. The materials may hence be used for pulsatile drug release into the stomach in the fed state, or in elderly patients.

\section{Acknowledgements}

The authors would like to thank the EPSRC for funding access to the TEM instruments in Oxford Materials under the Materials Equipment Access scheme (grant reference: EP/F01919X/1), to the SEM facility at the Nanovision Centre (QMUL), and to the National EPSRC XPS User Service (NEXUS) at Newcastle University. We also thank Mr David McCarthy for recording SEM images. 


\section{References}

1 G. R. Williams, N. P. Chatterton, T. Nazir, D.-G. Yu, L.-M. Zhu and C. J. Branford-White, Ther. Delivery, 2012, 3, 515-533.

2 Z.-M. Huang, Y. Z. Zhang, M. Kotaki and S. Ramakrishna, Compos. Sci. Technol., 2003, 63, 2223-2253.

3 S. Chakraborty, I. C. Liao, A. Adler and K. W. Leong, Adv. Drug Delivery Rev., 2009, 61, 1043-1054.

4 C. J. Luo, S. D. Stoyanov, E. Stride, E. Pelan and M. Edirisinghe, Chem. Soc. Rev., 2012, 41, 4708-4735.

5 X. Shen, D. Yu, L. Zhu, C. Branford-White, K. White and N. P. Chatterton, Int. J. Pharm., 2011, 408, 200-207.

6 X. M. Wu, C. J. Branford-White, L. M. Zhu, N. P. Chatterton and D. G. Yu, J. Mater. Sci.: Mater. Med., 2010, 21, 2403-2411.

7 D.-G. Yu, X.-F. Zhang, X.-X. Shen, C. Brandford-White and L.-M. Zhu, Polym. Int., 2009, 58, 1010-1013.

8 W. G. Cui, X. H. Li, X. L. Zhu, G. Yu, S. B. Zhou and J. Weng, Biomacromolecules, 2006, 7, 1623-1629.

9 G. Verreck, I. Chun, J. Rosenblatt, J. Peeters, A. V. Dijck, J. Mensch, M. Noppe and M. E. Brewster, J. Controlled Release, 2003, 92, 349-360.

10 Y.-N. Jiang, H.-Y. Mo and D.-G. Yu, Int. J. Pharm., 2012, 438, 232-239.

11 D. G. Yu, G. R. Williams, X. Wang, X. K. Liu, H. L. Li and S. W. A. Bligh, RSC Adv., 2013, 3, 4652-4658.

12 M. H. El-Newehy, S. S. Al-Deyab, E.-R. Kenawy and A. AbdelMegeed, Fibers Polym., 2012, 13, 709-717.

13 Z. X. Meng, W. Zheng, L. Li and Y. F. Zheng, Mater. Chem. Phys., 2011, 125, 606-611.

14 S. S. Sundar and D. Sangeetha, J. Mater. Sci.: Mater. Med., 2012, 23, 1421-1430.

15 T. Bussemer, I. Otto and R. Bodmeier, Crit. Rev. Ther. Drug Carrier Syst., 2001, 18, 433-458.

16 L. E. Kalantzi, E. Karavos, E. X. Koutris and D. N. Bikiaris, Recent Pat. Drug Delivery Formulation, 2009, 3, 49-63.
17 C. D. Saquing, C. Tang, B. Monian, C. A. Bonino, J. L. Manasco, E. Alsberg and S. A. Khan, Ind. Eng. Chem. Res., 2013, 52, 8692-8704.

18 H. Nie, A. He, W. Wu, J. Zheng, S. Xu, J. Li and C. C. Han, Polymer, 2009, 50, 4926-4934.

19 S. Moon, B.-y. Ryu, J. Choi, B. Jo and R. J. Farris, Polym. Eng. Sci., 2009, 53-59.

20 R. Rošic, J. Pelipenko, P. Kocbek, S. Baumgartner, M. Bešter-Rogač and J. Kristl, Eur. Polym. J., 2012, 48, 1374-1384.

21 C. A. Bonino, M. D. Krebs, C. D. Saquing, S. I. Jeong, K. L. Shearer, E. Alsberg and S. A. Khan, Carbohydr. Polym., 2011, 85, 111-119.

22 S. Alborzi, L.-T. Lim and Y. Kakuda, J. Microencapsulation, 2013, 30, 64-71.

23 J. S. Im, S. K. Lee, B. C. Bai and Y.-S. Lee, J. Ind. Eng. Chem., 2012, 18, 325-330.

24 C. a. Bonino, K. Efimenko, S. I. Jeong, M. D. Krebs, E. Alsberg and S. a. Khan, Small, 2012, 8, 1928-1936.

25 G. Ma, D. Fang, Y. Liu, X. Zhu and J. Nie, Carbohydr. Polym., 2012, 87, 737-743.

26 L. Maggi, L. Segale, M. L. Torre, E. O. Machiste and U. Conte, Biomaterials, 2002, 23, 1113-1119.

27 J. Balasubramaniam, V. U. Rao, M. Vasudha, J. Babu and P. S. Rajinikanth, Curr. Drug Delivery, 2007, 4, 249-256.

28 Z. Rahman, K. Kohli, S.-Q. Zhang, R. K. Khar, M. Ali, N. A. Charoo, M. Tauseef, A. A. A. Shamsher, N. N. Mohammed and M. A. Repka, J. Pharm. Pharmacol., 2008, 60, 615-623.

29 P. S. Rajinikanth, C. Sankar and B. Mishra, Drug Delivery, 2003, 10, 21-28.

30 Y. Cao, X. Shen, J. Guom, Q. Chen and X. Jiang, Biomacromolecules, 2005, 6, 2189-2196.

31 M. Huguet, A. Groboillot, R. Nueffid, D. Poncelelt and E. Dellacherie, J. Appl. Polym. Sci., 1994, 51, 1427-1432. 